\title{
On the uniqueness of the solution of a boundary-value problem for weakly nonlinear integro-differential equations with parameters
}

\author{
Olga B. Nesterenko
}

Presented by A. M. Samoilenko

\begin{abstract}
The conditions of existence of the unique solution of a boundary-value problem for weakly nonlinear integro-differential equations with parameters and the equivalence of such problem and the appropriate integral equation are established.
\end{abstract}

Keywords. Boundary-value problem, integro-differential equation, uniqueness of a solution.

\section{Introduction}

At the present time, the significant attention is paid to such branch of the theory of boundaryvalue problems as the study of solutions of the problems that include parameters in the equation or in boundary conditions. In particular, the work by N. I. Ronto and V. A. Ronto [14] was devoted to the questions of the existence and the construction of solutions of the boundary-value problems in the case where a parameter enters the boundary conditions. The monograph by A. A. Boichuk, V. F. Zhuravlev, and A. M. Samoilenko [1] has clarified the approach to the study of Noetherian problems including the problems with limitations. The boundary-value problems with parameters for multifrequency oscillatory systems and impulsive oscillatory systems with were studied with the help of the method of averaging in works by A. M. Samoilenko, R. I. Petryshyn, and L. M. Lakusta $[10,15]$. Works $[13,14]$ have established the conditions of existence of solutions of the boundary-value problems with parameters with the use of approximation methods. The conditions of solvability of the singular integral equations with parameters and limitations were considered in the work by O. B. Polishchuk [11]. In the works by A. Yu. Luchka [5-7], O. I. Kovtun [4], O. B. Polishchuk [12], and V. A. Feruk [2,3,7], the investigation of problems with limitations were carried out with the help of problems with parameters. In [9], the author has established the conditions of existence of solutions of a boundary-value problem for the linear integro-differential equations with limitations and has substantiated the application of the iteration method to them. The present work considers the questions of existence and uniqueness of the solution of a boundary-value problem for weakly nonlinear integro-differential equations with parameters. While solving these questions, the method developed in [5-9] is used.

\section{Statement of the problem}

We consider the integro-differential equation

$$
(L x)(t)=f(t)+C(t) \lambda+\varepsilon \int_{a}^{b} H(t, s) F\left(s, x(s), x^{\prime}(s), \ldots, x^{(m-1)}(s)\right) d s
$$

Translated from Ukrains'kiı̆ Matematychnyı̆ Visnyk, Vol. 9, No. 4, pp. 568-577, October-November, 2012. Original article submitted September 26, 2012 
and pose the problem of determination of a function $x \in W_{2}^{m}[a, b]$ and a parameter $\lambda \in \mathbb{R}^{l}$ such that they satisfy Eq. (1.1) almost everywhere, boundary conditions, and the limitation

$$
U(x)=\gamma, \quad \int_{a}^{b} S(t) x(t) d t=\alpha .
$$

If such pair $(x(t), \lambda)$ exists, then problem (1.1), (1.2) is considered compatible.

In representation (1.1) and (1.2),

$$
(L x)(t)=x^{(m)}(t)+p_{1}(t) x^{(m-1)}(t)+\cdots+p_{m}(t) x(t),
$$

$t \in[a, b], \varepsilon$ is a sufficiently small nonnegative parameter, $f \in L_{2}[a, b],\left\{p_{1}, \ldots, p_{m}\right\} \subset L_{2}[a, b]$, the kernel $H(t, s)$ is square-summable in the totality of variables, and the $(1 \times l)$-matrix $C(t)$ and $(l \times 1)$ matrix $S(t)$ whose elements are linearly independent functions square-summable on the interval $[a, b]$, the constant $(m \times 1)$-matrix $U$ whose elements take the form

$$
U_{\nu}(x)=\sum_{i=1}^{m}\left(\alpha_{\nu i} x^{(i-1)}(a)+\beta_{\nu i} x^{(i-1)}(b)\right),
$$

$\gamma \in \mathbb{R}^{m}$, and $\alpha \in \mathbb{R}^{l}$ are given.

We consider also that the operator

$$
(F x)(t)=F\left(t, x(t), x^{\prime}(t), \ldots, x^{(m-1)}(t)\right)
$$

maps the space $W_{2}^{m}[a, b]$ onto the space $L_{2}[a, b]$. The function $F:[a, b] \times \mathbb{R}^{m} \rightarrow \mathbb{R}$, which generates it, satisfies the Lipschitz condition

$$
\left|F\left(t, u_{0}, \ldots, u_{m-1}\right)-F\left(t, v_{0}, \ldots, v_{m-1}\right)\right| \leq \sum_{i=0}^{m-1} \tau_{i}\left|u_{i}-v_{i}\right|,
$$

for any $\left\{u_{i}, v_{i}\right\} \subset \mathbb{R}, \tau_{i} \in \mathbb{R}^{+}, i=\overline{0, m-1}$.

Using the method developed in [5-9], we will show that problem (1.1), (1.2) is equivalent to an integral equation without limitations. Let us consider the generating problem

$$
\begin{gathered}
(A x)(t)=C(t) \lambda+y(t), \quad U(x)=\gamma, \\
\int_{a}^{b} S(t) x(t) d t=\alpha,
\end{gathered}
$$

where

$$
(A x)(t)=x^{(m)}(t)+c_{1}(t) x^{(m-1)}(t)+\cdots+c_{m}(t) x(t),
$$

and the given function $y \in L_{2}[a, b]$ and the coefficients $c_{1}(t), \ldots, c_{m}(t)$ are continuous on the interval $[a, b]$.

In [9], it was proved that, in the case where the homogeneous problem

$$
(A x)(t)=C(t) \lambda, \quad U(x)=0, \quad \int_{a}^{b} S(t) x(t) d t=0
$$


has only the trivial solution, there exist a vector $\sigma \in \mathbb{R}^{l}$, functions $h(t)$ and $G(t, s)$, and a $(l \times 1)$-matrix $\Gamma(s)$ such that the unique solution of the inhomogeneous problem (1.5), (1.6) is given by the formulas

$$
x(t)=h(t)+\int_{a}^{b} G(t, s) y(s) d s, \quad \lambda=\sigma+\int_{a}^{b} \Gamma(s) y(s) d s .
$$

We write Eq. (1.1) as

$$
\begin{aligned}
& x^{(m)}(t)+c_{1}(t) x^{(m-1)}(t)+\cdots+c_{m}(t) x(t) \\
& =x^{(m)}(t)+c_{1}(t) x^{(m-1)}(t)+\cdots+c_{m}(t) x(t)-x^{(m)}(t)-p_{1}(t) x^{(m-1)}(t)-\cdots-p_{m}(t) x(t)+f(t)+C(t) \lambda \\
& +\varepsilon \int_{a}^{b} H(t, s) F\left(s, x(s), x^{\prime}(s), \ldots, x^{(m-1)}(s)\right) d s .
\end{aligned}
$$

Introducing the notation

$$
\begin{gathered}
r_{k}(t)=c_{k}(t)-p_{k}(t), \quad k=\overline{1, m}, \\
y(t)=f(t)+r_{1}(t) x^{(m-1)}(t)+\cdots+r_{m}(t) x(t)+\varepsilon \int_{a}^{b} H(t, s) F\left(s, x(s), x^{\prime}(s), \ldots, x^{(m-1)}(s)\right) d s
\end{gathered}
$$

and using formula (1.7), we write relation (1.10) as

$$
(A x)(t)=C(t) \lambda+y(t) .
$$

Hence, problem (1.1), (1.2) becomes problem (1.5), (1.6). With regard for the homogeneous problem (1.8), the latter the unique solution given by formulas (1.9).

Substituting the relation in (1.9) in the right-hand side of relation (1.11), we obtain

$$
\begin{aligned}
y(t) & =f(t)+r_{1}(t) h^{(m-1)}(t)+\cdots+r_{m}(t) h(t)+\int_{a}^{b}\left(r_{1}(t) \frac{\partial^{m-1}}{\partial t^{m-1}} G(t, s)+\cdots+r_{m}(t) G(t, s)\right) y(s) d s \\
& +\varepsilon \int_{a}^{b} H(t, s) F\left(s, h(s)+\int_{a}^{b} G(s, \xi) y(\xi) d \xi, \ldots, h^{(m-1)}(s)+\int_{a}^{b} \frac{\partial^{m-1}}{\partial s^{m-1}} G(s, \xi) y(\xi) d \xi\right) d s . \quad(1.12)
\end{aligned}
$$

Let us take into account that

$$
(A x)(t)-(L x)(t)=(B x)(t)=r_{1}(t) h^{(m-1)}(t)+\cdots+r_{m}(t) x(t)
$$

and denote

$$
\begin{aligned}
& K(t, s)=(B G)(t, s)=r_{1}(t) \frac{\partial^{m-1}}{\partial t^{m-1}} G(t, s)+\cdots+r_{m}(t) G(t, s), \\
& g(t)=f(t)+(B h)(t)=f(t)+r_{1}(t) h^{(m-1)}(t)+\cdots+r_{m}(t) h(t) .
\end{aligned}
$$

Now, the integral equation (1.12) takes the form

$$
\begin{aligned}
y(t) & =g(t)+\int_{a}^{b} K(t, s) y(s) d s \\
& +\varepsilon \int_{a}^{b} H(t, s) F\left(s, h(s)+\int_{a}^{b} G(s, \xi) y(\xi) d \xi, \ldots, h^{(m-1)}(s)+\int_{a}^{b} \frac{\partial^{m-1}}{\partial s^{m-1}} G(s, \xi) y(\xi) d \xi\right) d s .
\end{aligned}
$$


By using a simple reasoning, we can establish that problem (1.1), (1.2) is equivalent to the integral equation (1.15). The equivalence means that if $y^{*} \in L_{2}[a, b]$ is a solution of Eq. (1.15), then $\left(x^{*}(t), \lambda^{*}\right)$ is a solution of the boundary-value problem (1.1), (1.2), and

$$
x^{*}(t)=h(t)+\int_{a}^{b} G(t, s) y^{*}(s) d s, \quad \lambda^{*}=\sigma+\int_{a}^{b} \Gamma(s) y^{*}(s) d s .
$$

On the contrary, if $\left(x^{*}(t), \lambda^{*}\right)$ is a solution of problem (1.1), (1.2), then the function

$$
y^{*}(t)=\left(A x^{*}\right)(t)-C(t) \lambda^{*}
$$

is a solution of Eq. (1.15).

Indeed, let $y^{*}(t)$ be a solution of the integral equation (1.15). Then the function $x^{*}(t)$ and the parameter $\lambda^{*}$ that are defined by formulas (1.16) are a solution of problem (1.1), (1.2).

Since the validity of the equalities

$$
\left(A x^{*}\right)(t)=C(t) \lambda^{*}+y^{*}(t), \quad U\left(x^{*}\right)=\gamma, \quad \int_{a}^{b} S(t) x^{*}(t) d t=\alpha
$$

can be easily verified, the boundary conditions (1.2) are obviously satisfied. We now establish that the function $x^{*}(t)$ satisfies Eq. (1.1). For this purpose, we use formulas (1.13), (1.18), (1.16), and (1.14) and obtain

$$
\begin{aligned}
f(t)+C(t) \lambda^{*}-\left(L x^{*}\right)(t)=f(t)+C(t) \lambda^{*}-\left(A x^{*}\right)(t)+\left(B x^{*}\right)(t) & \\
=f(t)-y^{*}(t)+r_{1}(t)\left(h(t)+\int_{a}^{b} G(t, s) y^{*}(s) d s\right)^{(m-1)} & +\cdots+r_{m}(t)\left(h(t)+\int_{a}^{b} G(t, s) y^{*}(s) d s\right) \\
& =g(t)-y^{*}(t)+\int_{a}^{b} K(t, s) y^{*}(s) d s
\end{aligned}
$$

Hence, taking formulas (1.19) and (1.16) into account, we have finally:

$$
\begin{aligned}
f(t)+ & C(t) \lambda^{*}-\left(L x^{*}\right)(t) \\
& +\varepsilon \int_{a}^{b} H(t, s) F\left(s, x^{*}(s), \ldots, x^{*(m-1)}(s)\right) d s=g(t)-y^{*}(t)+\int_{a}^{b} K(t, s) y^{*}(s) d s \\
& +\varepsilon \int_{a}^{b} H(t, s) F\left(s, h(s)+\int_{a}^{b} G(s, \xi) y^{*}(\xi) d \xi, \ldots, \frac{d^{m-1}}{d s^{m-1}}\left(h(s)+\int_{a}^{b} G(s, \xi) y^{*}(\xi) d \xi\right)\right) d s=0 .
\end{aligned}
$$

Indeed, problem (1.1), (1.2) has the solution $\left(x^{*}(t), \lambda^{*}\right)$, which is uniquely determined by formulas (1.16) and by the known solution $y^{*}(t)$ of the integral equation (1.15).

On the contrary, let $\left(x^{*}(t), \lambda^{*}\right)$ be a solution of problem (1.1), (1.2). By definition, the function $x^{*}(t)$ belongs to the class $W_{2}^{m}[a, b]$ and satisfies conditions (1.2). Therefore, as was established above, the equality

$$
x^{*}(t)=h(t)+\int_{a}^{b} G(t, s)\left(A x^{*}\right)(s) d s
$$

holds. 
We now show that the function $y^{*}(t)$ that is defined by formula (1.17) is a solution of the integral equation (1.15). On the basis of formulas (1.17) and (1.20), we have

$$
h(t)+\int_{a}^{b} G(t, s) y^{*}(s) d s=h(t)+\int_{a}^{b} G(t, s)\left(A x^{*}\right)(s) d s=x^{*}(t) .
$$

Using definition (1.14) and expression (1.21), we obtain

$$
\begin{gathered}
g(t)+\int_{a}^{b} K(t, s) y^{*}(s) d s=f(t)+(B h)(t)+\int_{a}^{b}(B G)(t, s) y^{*}(s) d s=f(t)+\left(B x^{*}\right)(t), \\
F\left(s, h(s)+\int_{a}^{b} G(s, \xi) y^{*}(\xi) d \xi, \ldots, \frac{d^{m-1}}{d s^{m-1}}\left(h(s)+\int_{a}^{b} G(s, \xi) y^{*}(\xi) d \xi\right)\right) \\
=F\left(s, x^{*}(s), \ldots, \frac{d^{m-1}}{d s^{m-1}} x^{*}(s)\right) .
\end{gathered}
$$

Substituting relation (1.17) in Eq. (1.15) with regard for formulas (1.22), (1.23), and (1.13), we have finally

$$
\begin{aligned}
& g(t)-y^{*}(s)+\int_{a}^{b} K(t, s) y^{*}(s) d s \\
& \quad+\varepsilon \int_{a}^{b} H(t, s) F\left(s, h(s)+\int_{a}^{b} G(s, \xi) y^{*}(\xi) d \xi, \ldots, \frac{d^{m-1}}{d s^{m-1}}\left(h(s)+\int_{a}^{b} G(s, \xi) y^{*}(\xi) d \xi\right)\right) d s \\
& \quad=f(t)-\left(L x^{*}\right)(t)+C(t) \lambda^{*}+\varepsilon \int_{a}^{b} H(t, s) F\left(s, x^{*}(s), \ldots, \frac{d^{m-1}}{d s^{m-1}} x^{*}(s)\right) d s=0,
\end{aligned}
$$

since $\left(x^{*}(t), \lambda^{*}\right)$ is a solution of Eq. (1.1). Equality (1.24) testifies that, indeed, the function $y^{*}(t)$ given by formula (1.17) is a solution of the integral equation (1.15).

Theorem 1.1 ([8]). If the auxiliary problem (1.5), (1.6) has the unique solution, then problem (1.1), (1.2) is compatible if and only if there exists a solution of Eq. (1.15).

Theorem 1.2. If the generating problem (1.5), (1.6) has the unique solution, then the unique solution of problem (1.1), (1.2) exists only if the integral equation (1.15) has the unique solution.

Proof. Let $y^{*}(t)$ be the unique solution of the integral equation (1.15). Then, by Theorem 1.1, there exists a solution of problem (1.1), (1.2) that is defined by formulas (1.9).

Assume that problem (1.1), (1.2) has another solution $(\bar{x}(t), \bar{\lambda})$ different from the first one $\left(x^{*}(t), \lambda^{*}\right)$, i.e., $\bar{x}(t) \neq x^{*}(t), \bar{\lambda} \neq \lambda^{*}$, or at least one of these inequalities is satisfied.

Then, by Theorem 1.1, the function

$$
\bar{y}(t)=(A \bar{x})(t)-C(t) \bar{\lambda}
$$


is a solution of the integral equation (1.15). The validity of the equality

$$
y^{*}(t)-\bar{y}(t)=\left(A x^{*}-A \bar{x}\right)(t)+C(t)\left(\bar{\lambda}-\lambda^{*}\right)
$$

follows from (1.17) and (1.25). By assumption, the integral equation (1.15) has only a single solution, i.e., $y^{*}(t)=\bar{y}(t)$. Let us introduce the notation $u(t)=x^{*}(t)-\bar{x}(t), \mu=\lambda^{*}-\bar{\lambda}$. With regard for relation (1.26) and the fact that $x^{*}(t)$ and $\bar{x}(t)$ satisfy the same boundary conditions, we obtain

$$
(A u)(t)=C(t) \mu, \quad U(u)=0, \quad \int_{a}^{b} S(t) u(t) d t=0 .
$$

Equalities (1.27) hold only if $u(t)=0, \mu=0$. Hence, $x^{*}(t)=\bar{x}(t)$ and $\lambda^{*}=\bar{\lambda}$. But this contradicts the assumption. Thus, if the integral equation has the unique solution, there exists only the unique solution of problem (1.1), (1.2).

On the contrary, let it be known that problem $(1.1),(1.2)$ has the unique solution $\left(x^{*}(t), \lambda^{*}\right)$. By Theorem 1.1, the function $y^{*}(t)$ defined by formula (1.17) is a solution of the integral equation (1.15). Moreover, it is easy to verify the validity of the formulas

$$
\begin{gathered}
x^{*}(t)=h(t)+\int_{a}^{b} G(t, s)\left(\left(A x^{*}\right)(s)-C(s) \lambda^{*}\right) d s, \\
\lambda^{*}=\sigma+\int_{a}^{b} \Gamma(s)\left(\left(A x^{*}\right)(s)-C(s) \lambda^{*}\right) d s=0 .
\end{gathered}
$$

Assume that there exists one more solution $\bar{y}(t)$ of the integral equation (1.15). Let it be such that $\bar{y}(t) \neq y^{*}(t)$. Then problem (1.1), (1.2) has one more solution

$$
\bar{x}(t)=h(t)+\int_{a}^{b} G(t, s) \bar{y}(s) d s, \quad \bar{\lambda}=\sigma+\int_{a}^{b} \Gamma(s) \bar{y}(s) d s .
$$

On the basis of formulas (1.28), (1.29), (1.17), and (1.30), we obtain

$$
\begin{gathered}
x^{*}(t)-\bar{x}(t)=\int_{a}^{b} G(t, s)\left(y^{*}(s)-\bar{y}(s)\right) d s, \\
\lambda^{*}-\bar{\lambda}=\int_{a}^{b} \Gamma(s)\left(y^{*}(s)-\bar{y}(s)\right) d s .
\end{gathered}
$$

Since problem (1.1), (1.2) has only one solution, we have $x^{*}(t)=\bar{x}(t)$ and $\lambda^{*}=\bar{\lambda}$. Therefore, equalities (1.31), (1.32) take the form

$$
\int_{a}^{b} G(t, s)\left(y^{*}(s)-\bar{y}(s)\right) d s=0, \quad \int_{a}^{b} \Gamma(s)\left(y^{*}(s)-\bar{y}(s)\right) d s=0 .
$$

Equalities (1.33) hold only in the case where $y^{*}(t)=\bar{y}(t)$, which contradicts the assumption. Thus, the uniqueness of the solution of problem (1.1), (1.2) implies the uniqueness of the solution of the integral equation (1.15). 


\section{REFERENCES}

1. A. A. Boichuk, V. F. Zhuravlev, and A. M. Samoilenko, Generalized Inverse Operators and Noetherian Boundary-Value Problems [in Russian], Institute of Mathematics of the NAS of Ukraine, Kiev, 1995.

2. V. A. Feruk, "The iteration method for systems of nonlinear differential equations with delay and limitations," Nelin. Kolyv., 6, No. 3, 428-436 (2003).

3. V. A. Feruk, "A version of the projection-iteration method for systems of linear differential equations with delay of the neutral type and limitations," Nelin. Kolyv., 9, No. 4, 564-573 (2006).

4. O. I. Kovtun, "Projection-iteration methods for integral equations with weak nonlinearity and additional conditions," Nelin. Kolyv., 3, No. 3, 365-374 (2000).

5. A. Yu. Luchka, "Integral equations with limitations and methods of their solution," Kibern. Sist. Anal., No. 3, 82-96 (1996).

6. A. Yu. Luchka and O. M. Voznyuk, "The iteration method for integral equations with limitations," Nelin. Kolyv., 5, No. 3, 179-192 (2002).

7. A. Yu. Luchka and V. A. Feruk, "A modified projection-iteration method for systems of quasilinear differential equations with delay and limitation," Nelin. Kolyv., 7, No. 2, 188-207 (2004).

8. A. Yu. Luchka and O. B. Nesterenko, "Methods of solution of boundary-value problems for weakly nonlinear integro-differential equations with parameters and limitations," Ukr. Mat. Zh., 61, No. 5, 672-679 (2009).

9. O. B. Nesterenko, "The iteration method of solution of integro-differential equations with limitations," Nelin. Kolyv., 10, No. 3, 336-347 (2007).

10. R. I. Petryshyn and L. M. Lakusta, "Evaluation of the error of the method of averaging in impulsive boundary-value problems with parameters," Nelin. Kolyv., 5, No. 2, 193-200 (2002).

11. O. B. Polishchuk, "A modified projection-iteration method of solution of singular integral equations with parameters and with small nonlinearity," Ukr. Mat. Zh., 51, No. 3, 418-423 (1999).

12. O. B. Polishchuk, "The conditions of compatibility of a problem with limitations for singular integral equations," Nelin. Kolyv., 3, No. 4, 511-514 (2000).

13. N. I. Ronto, "Some exact solutions of solvability of the initial problem for systems of linear functionaldifferential equations," Nelin. Kolyv., 7, No. 4, 538-554 (2004).

14. N. I. Ronto and V. A. Ronto, "On a method of study of boundary-value problems with parameters," in Boundary-Value Problems of Mathematical Physics [in Russian], Naukova Dumka, Kiev, 1990, pp. 3-10.

15. A. M. Samoilenko, R. I. Petryshyn, and L. M. Lakusta, "The averaging of boundary-value problems with parameters for multipoint impulsive systems," Ukr. Mat. Zh., 54, No. 9, 1237-1249 (2002).

Translated from Ukrainian by V. V. Kukhtin

\section{Olga B. Nesterenko}

Kyiv National University of Technologies and Design, 2, Nemyrovych-Danchenko Str., Kyiv 01011, Ukraine

E-Mail: Olga_kiev@mail.ru 